\title{
Efficacy, tolerability, and safety of erenumab for the preventive treatment of persistent post-traumatic headache attributed to mild traumatic brain injury: an open-label study
}

Håkan Ashina', Afrim Iljazi ${ }^{1}$, Haidar Muhsen Al-Khazali', Anna Kristina Eigenbrodt ${ }^{1}$, Eigil Lindekilde Larsen ${ }^{1}$, Amalie Middelboe Andersen ${ }^{1}$, Kevin John Hansen ${ }^{1}$, Karoline Bendix Bräuner ${ }^{1}$, Thomas Mørch-Jessen', Basit Chaudhry ${ }^{1}$, Sonja Antic ${ }^{2}$, Casper Emil Christensen ${ }^{1}$, Messoud Ashina', Faisal Mohammad Amin ${ }^{1}$ and Henrik Winther Schytz ${ }^{*}$

\begin{abstract}
Background: Calcitonin gene-related peptide (CGRP) has recently been implicated in the pathogenesis of posttraumatic headache (PTH), which raises the prospect for therapeutic use of monoclonal antibodies targeting CGRP or its receptor. Therefore, we decided to assess the efficacy, tolerability, and safety of erenumab for prevention of persistent PTH attributed to mild traumatic brain injury.

Methods: A single-center, non-randomized, single-arm, open-label study of erenumab for adults aged 18-65 years with persistent PTH. Patients were assigned to receive 140-mg erenumab monthly by two subcutaneous 1-mL injections, given every 4 weeks for 12 weeks. The primary outcome measure was the mean change in number of monthly headache days of moderate to severe intensity from baseline (4-week pretreatment period) to week 9 through 12. Tolerability and safety endpoints were adverse events (i.e. number and type).

Results: Eighty-nine of 100 patients completed the open-label trial. At baseline, the mean monthly number of headache days of moderate to severe intensity was 15.7. By week 9 through 12, the number was reduced by 2.8 days. The most common adverse events were constipation $(n=30)$ and injection-site reactions $(n=15)$. Of 100 patients who received at least one dose of erenumab, two patients discontinued the treatment regimen due to adverse events.

\footnotetext{
* Correspondence: henrik.winther.schytz.01@regionh.dk

'Danish Headache Center, Department of Neurology, Rigshospitalet Glostrup, Faculty of Health and Medical Sciences, University of Copenhagen, Valdemar Hansens Vej 5, DK-2600 Glostrup, Denmark

Full list of author information is available at the end of the article
}

C C The Author(s). 2020 Open Access This article is licensed under a Creative Commons Attribution 4.0 International License, which permits use, sharing, adaptation, distribution and reproduction in any medium or format, as long as you give appropriate credit to the original author(s) and the source, provide a link to the Creative Commons licence, and indicate if changes were made. The images or other third party material in this article are included in the article's Creative Commons licence, unless indicated otherwise in a credit line to the material. If material is not included in the article's Creative Commons licence and your intended use is not permitted by statutory regulation or exceeds the permitted use, you will need to obtain permission directly from the copyright holder. To view a copy of this licence, visit http://creativecommons.org/licenses/by/4.0/ The Creative Commons Public Domain Dedication waiver (http://creativecommons.org/publicdomain/zero/1.0/) applies to the data made available in this article, unless otherwise stated in a credit line to the data. 
(Continued from previous page)

Conclusions: Among patients with persistent PTH, erenumab resulted in a lower frequency of moderate to severe headache days in this 12-week open-label trial. In addition, erenumab was well-tolerated as discontinuations due to adverse events were low. Placebo-controlled randomized clinical trials are needed to adequately evaluate the efficacy and safety of erenumab in patients with persistent PTH.

Trial registration: ClinicalTrials.Gov, NCT03974360. Registered on April 17, 2019 - Retrospectively registered

Keywords: Concussion, Secondary headache, Head trauma, Head injury, Clinical management

\section{Introduction}

Post-traumatic headache $(\mathrm{PTH})$ is a common sequela of mild traumatic brain injury (TBI) $[1,2]$, with a lifetime prevalence of $4.7 \%$ in men and $2.4 \%$ in women [3]. In addition, it has been documented that symptoms suggestive of depression, sleep disturbances, and post-traumatic stress disorder are frequent following mild TBI [2, 4, 5], including in those who develop persistent PTH [6, 7]. A nationwide registry-based study has also found an increased suicide risk in individuals with TBI, compared with the general population without TBI [8]. Despite the widespread prevalence and disability associated with PTH [4], there is little evidence to support any acute or preventive medication therapy [5]. In fact, no pharmacological agent has been approved for the treatment of PTH. As such, clinicians often choose a preventive treatment based on the individual patients' headache phenotype. This approach has not been systematically investigated and, thus, lacks evidence. Taken together, there remains a considerable unmet need for mechanism-based treatments that are effective and well-tolerated. In this context, monoclonal antibodies targeting calcitonin gene-related peptide (CGRP) or its receptor might hold great promise as PTH often mimics a migraine-like headache [6] and anti-CGRP monoclonal antibodies have proven effective for preventive treatment of migraine [7-11]. In addition, preclinical data have emerged and demonstrated hypersensitivity to CGRP in concussed rodents $[12,13]$. Thus, we find it timely to assess the efficacy, tolerability, and safety of erenumab for preventive treatment of persistent PTH attributed to mild TBI.

\section{Methods}

\section{Study oversight}

This trial was approved by the Regional Health Research Ethics Committee of the Capital Region of Denmark (identifier: H-18050498). In addition, study approval was also obtained from the Danish Medicines Agency (identifier: 2018-1104) and the Danish Data Protection Agency (identifier: VD-2019-20). Written informed consent was obtained from each participant before any study procedures or assessments were performed. Moreover, this trial was conducted in accordance with the Declaration of Helsinki [14].

\section{Study participants}

Patients were recruited from the outpatient clinic of the Danish Headache Center and from neurological departments and rehabilitation centers in the Capital Region of Denmark as well as the Region of Southern Denmark. Patients included males and females aged 18 to 65 years with a history of persistent headache attributed to mild TBI in accordance with the 3rd edition of the International Classification of Headache Disorders (ICHD-3), [15]. Patients were allowed to use one concomitant preventive headache medication taken at a stable dose, i.e. no changes to the dose within 2 months before the baseline phase. In addition, patients were required to maintain stable dosing during the baseline phase and throughout the treatment phase. Use of acute headache medications was permitted, although patients with medication-overuse headache were excluded. Patients were also excluded if they had any history of primary headache disorder, except infrequent tension-type headache (TTH), or any history of whiplash injury. The complete list of inclusion and exclusion criteria is available in Supplement 1.

\section{Study design and procedures}

This non-randomized, single-arm clinical trial consisted of a screening phase ( 0 to 2 weeks), baseline phase (4 weeks), and an open-label treatment phase (12 weeks). The patients had five scheduled study site visits: screening, baseline (dose 1 ), week 4 (dose 2 ), week 8 (dose 3 ), and week 12 (final evaluation). During the screening phase, site investigators contacted all potential participants by phone to assess eligibility for study inclusion.

At the screening visit, eligible participants signed the informed consent form and then underwent a thorough medical examination. An in-person semi-structured interview was performed by site investigators to record data on demographics, medical history, and full clinical course. In addition, the following study procedures were performed: electrocardiography, pregnancy testing, and blood sampling. Patients were instructed to complete a 4-week headache diary in paper format to establish headache characteristics and medication use (available in Supplement 2). At least $80 \%$ headache diary compliance was required to enter the open-label treatment phase. 
During the open-label treatment phase, patients received $140-\mathrm{mg}$ erenumab monthly by two subcutaneous 1 - $\mathrm{mL}$ injections at study visits on baseline (day 1 ), week 4 , and week 8 . For efficacy and safety assessments, patients were asked to record information daily using a headache diary in paper format. At the follow-up visits (weeks 4,8 , and 12), protocol-specified study procedures were performed, and site investigators assessed efficacy and safety as well as headache diary compliance. At least $80 \%$ compliance was required throughout the open-label treatment phase.

\section{Outcomes}

The primary outcome measure was the mean change in number of monthly headache days of moderate to severe intensity from baseline (4-week pretreatment period) to week 9-12. Secondary outcome measures of efficacy included 1) the mean change in number of monthly headache days of any intensity from baseline to week 9-12, 2) the proportion of patients achieving at least $50 \%$ reduction in the mean number of monthly headache days of any intensity from baseline to week 9-12,3) the proportion of patients achieving at least $25 \%$ reduction in the mean number of monthly headache days of any intensity from baseline to week 9-12,4) the proportion of patients achieving at least $75 \%$ reduction in the mean number of monthly headache days of any intensity from baseline to week $9-12,5)$ the mean change in disability score from baseline to week 12, as measured by the Headache Impact Test (HIT-6). Tolerability and safety endpoints were adverse events (i.e. number and type).

\section{Statistical analysis}

Efficacy outcomes measures were calculated based on headache diary entries and analyzed using a completecase analysis. The latter included patients who received all three doses of erenumab and had at least $80 \%$ compliance throughout the open-label treatment phase. The tolerability and safety analyses included all patients who received at least one dose of erenumab. Adverse events were tabulated as frequency counts. $\mathrm{R}$ statistical software version 3.6.0 was used to generate all data listing, summaries, and statistical analyses.

\section{Role of the funding source}

The trial was initiated by site investigators who were also responsible for data collection. All authors interpreted the data and contributed to the manuscript preparation, with support from employees of the study funder. Furthermore, all authors made the final decision to submit the manuscript for publication and attest to the accuracy and completeness of the data and reporting of adverse events. The study funder (Novartis Healthcare A/S) did not have the right to veto publication or to control the decision regarding to which journal the paper was submitted.

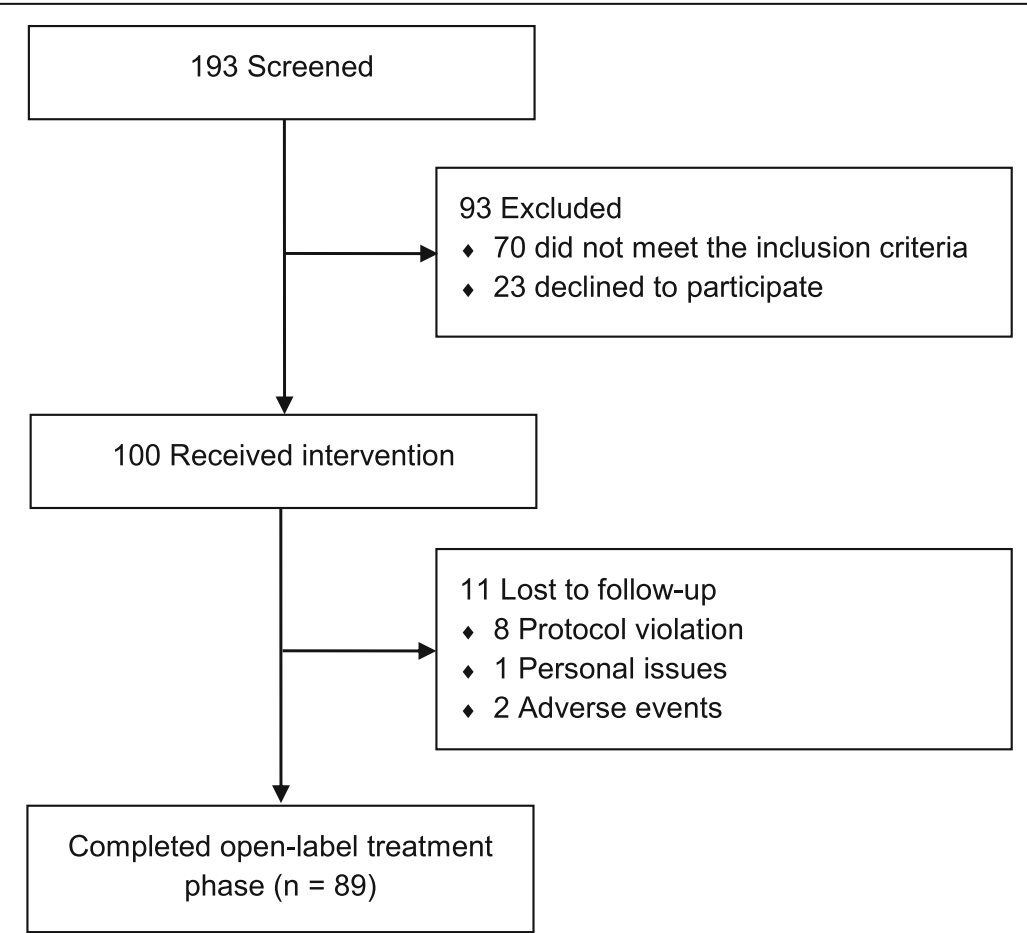

Fig. 1 Flow of Participants in an Open-Label Study of Erenumab for Prevention of Persistent Post-Traumatic Headache attributed to Mild Traumatic Brain Injury 
Table 1 Baseline Participant Characteristics

\begin{tabular}{lr}
\hline Characteristics & Pers \\
\hline Age, mean (SD), y & 35.1 .1 \\
Male/Female, \% & $25 / 7$ \\
Body Mass Index, mean (SD), kg/m² & 25.9 \\
Employment Status & 38 \\
Full-time employed, \% & 39 \\
Part-time employed, \% & 217 \\
Unemployed, \% & 2 \\
Retired, \% & 2
\end{tabular}

\section{Education}

Years of education, mean (SD), y Persistent PTH $(n=100)$

$35.1(11.3)$

No education besides completion of secondary school or high school, \%

Skilled labor, \%

Bachelor's degree, \%

Higher education, \%

Injury Cause

Fall, \%

Motor vehicle collision, \%

Sports-related injury, \%

Violence/assault, \%

Other unintentional injury, \%

\section{Disease History}

Time since mild traumatic brain injury, mean (SD), month

Current acute medication use, No.

Current preventive medication use, No.

History of preventive medication use, No.

No drug failures, $\%$

Failure of $\geq 1$ drug, $\%$

Failure of $\geq 2$ drugs, $\%$

Failure of $\geq 3$ drugs, \%

Failure of $\geq 4$ drugs, $\%$

Satisfaction with Current Treatment Status, \%

\section{Self-Rated Health}

Excellent, \%

Great, \%

Good, \%

Rather poor, \%

Poor, \%

Medico-Legal Issues / Litigation

Ongoing litigation, \%

Ended litigation, \%

Improvement in headache following end of litigation, No. (\%)

\section{Headache Phenotypes}

Chronic migraine-like, \%

Episodic migraine-like, \% 
Table 1 Baseline Participant Characteristics (Continued)

\begin{tabular}{ll}
\hline Characteristics & Persistent PTH $(\mathrm{n}=100)$ \\
\hline Episodic migraine-like combined with chronic TTH-like, \% & 27 \\
Episodic migraine-like combined with frequent TTH-like, \% & 6 \\
Chronic TTH-like, \% & 13 \\
Aura, \% & 11 \\
Family History of Primary Headache Disorders, \% & 31 \\
\hline
\end{tabular}

\section{Results}

\section{Study participants}

A total of 193 patients with persistent PTH were screened for eligibility (Fig. 1), with 23 patients who declined to participate and 70 patients who did not fulfill the eligibility criteria, mostly due to a history of whiplash injury, pre-trauma primary headache disorder, or medication-overuse headache. Thus, 100 patients (75 females and 25 males) were enrolled and received at least one dose of $140-\mathrm{mg}$ erenumab. Table 1 summarizes baseline demographics and clinical characteristics. The mean age (SD) was 35.1 (11.3) years while the mean body mass index (SD) was $25.9(5.3) \mathrm{kg} / \mathrm{m}^{2}$. In terms of employment status, $38 \%$ were full-time employed whereas $39 \%$ were part-time employed, $21 \%$ were unemployed, and lastly, $2 \%$ had retired from the workforce. The majority had either a bachelor's degree or higher education (58\%) while $14 \%$ had no education besides completion of secondary school or high school. Moreover, 39\% had ongoing litigation, whereas $39 \%$ as well had ended litigation. Lastly, 19\% had a history of pretrauma psychiatric illness.

The most common headache phenotypes were chronic migraine-like headache (53\%) followed by combined episodic migraine-like/TTH-like headache (34\%), and 'pure' chronic TTH-like headache (13\%). Current use of acute headache medication was reported by $89 \%$, whereas a history of preventive medication use was reported by $74 \%$. Of the latter, approximately one-fifth had failed at least four preventive medications. Overall, 79 of 100 patients were dissatisfied with their current treatment status.

A total of 89 of the 100 included patients completed the open-label treatment phase and provided data for the complete-case analysis of efficacy outcome measures. Of the 11 patients who did not complete the open-label treatment phase, eight patients were excluded due to protocol violations, i.e. lack of compliance with daily entries in the headache diary $(\mathrm{n}=4)$, logistical issues $(\mathrm{n}=3)$, and unwillingness to maintain stable dosing of a concomitant preventive medication during the treatment phase $(\mathrm{n}=1)$. In addition,

Table 2 Summary of Pre-Treatment Disease Characteristics and Outcome Measures

Persistent PTH $(n=89)$

\section{Disease Characteristics during 28-day Pre-Treatment Phase}

Headache days of any severity, mean (SD)

$24.6 \pm 6.1$

Headache days of moderate to severe intensity, mean (SD)

$15.7 \pm 9.6$

Days with use of any acute headache medication, mean (SD)

$4.0 \pm 4.4$

HIT-6 score, mean (SD)

$61.6 \pm 5.2$

Primary Outcome Measure

Mean change in number of monthly headache days of moderate to severe intensity from baseline to week 9-12 (SD)

Secondary Outcome Measures

Mean change in number of monthly headache days of any intensity from baseline to week 9-12 (SD)

$\geq 25 \%$ reduction in mean monthly headache days of any intensity, baseline to week $12, \%$

$\geq 50 \%$ reduction in mean monthly headache days of any intensity, baseline to week $12, \%$

$\geq 75 \%$ reduction in mean monthly headache days of any intensity, baseline to week $12, \%$

6

Mean change in HIT-6 score from baseline to week 12 (SD)

Post-Hoc Explorative Outcome Measures
$\geq 25 \%$ reduction in mean monthly headache days of moderate to severe intensity, baseline to week $12, \%$
47
$\geq 50 \%$ reduction in mean monthly headache days of moderate to severe intensity, baseline to week $12, \%$
28
$\geq 75 \%$ reduction in mean monthly headache days of moderate to severe intensity, baseline to week $12, \%$
13
Mean change in number of monthly days using acute headache medications, baseline to week 9-12 (SD)
$-0.4(5.2)$ 
another patient withdrew due to personal issues related to a stressful life events, while two patients were excluded due to adverse events ( $n=1$, worsened headache; $n=1$, dizziness).

\section{Efficacy}

At baseline, the mean number of headache days of moderate to severe intensity was $15.7 \pm 9.6$ days per month; by week 9 through 12, the number was reduced by $2.8 \pm$ 6.8 days (Table 2 ). A post-hoc analysis revealed that $\mathrm{a} \geq$ $50 \%$ reduction in the mean number of headache days of moderate to severe intensity was achieved for $28 \%$ of the patients (Fig. 2). Furthermore, $47 \%$ had achieved $\geq 25 \%$ reduction whereas $12 \%$ had achieved $\geq 75 \%$ reduction in mean number of headache days of moderate to severe intensity (Fig. 2). Moreover, patients were categorized for post-hoc subgroup analysis as follows: treatment-naïve patients $(\mathrm{n}=$ 24) and patients with at least 2 preventive treatment failures $(\mathrm{n}=36)$. In the former group, erenumab resulted in a reduction of $0.9 \pm 9.0$ headache days of moderate to severe intensity; by week 9 through 12 . The corresponding reduction was $3.0 \pm 5.2$ headache days of moderate to severe intensity in patients with at least 2 preventive treatment failures.

The mean number of headache days of any severity was $24.6 \pm 6.1$ days per month at baseline; by week 9 through 12 , the number was reduced by $1.7 \pm 6.9$ (Table 2 ). The $50 \%$ responder rate for headache days of any intensity was
$13 \%$ by week 9 through 12, whereas $21 \%$ achieved a $\geq 25 \%$ reduction and $6 \%$ achieved $\mathrm{a} \geq 75 \%$ reduction (Fig. 2 ).

At baseline, mean number of days per month with use of acute headache medication was $4.0 \pm 4.4$ (Table 2). By week 9 through 12, the number was reduced by 0.4 days per month. Mean HIT-6 scores were $61.6 \pm 5.2$ at baseline and $57.0 \pm 8.2$, yielding a reduction of 4.6 points (Table 2). Of 89 patients, $44 \%$ achieved a $\geq 5$-point reduction in HIT-6 score from baseline to week 9 through 12 .

\section{Tolerability and safety}

Overall, 100 patients received at least one dose of erenumab and were included in the tolerability and safety analyses (Table 3). Seventy-eight patients reported at least one adverse event, with the most common ones being constipation $(n=30)$ and injection-site reactions $(n=$ 15). Of the former, nine patients reported recurrent episodes of constipation. No serious adverse events were reported, although two patients experienced adverse events (dizziness and worsened headache) that led to treatment discontinuation.

\section{Discussion}

Treatment with 140-mg erenumab yielded a reduction of 2.8 headache days of moderate to severe intensity by week 9 through 12, while the rate of a $50 \%$ or greater

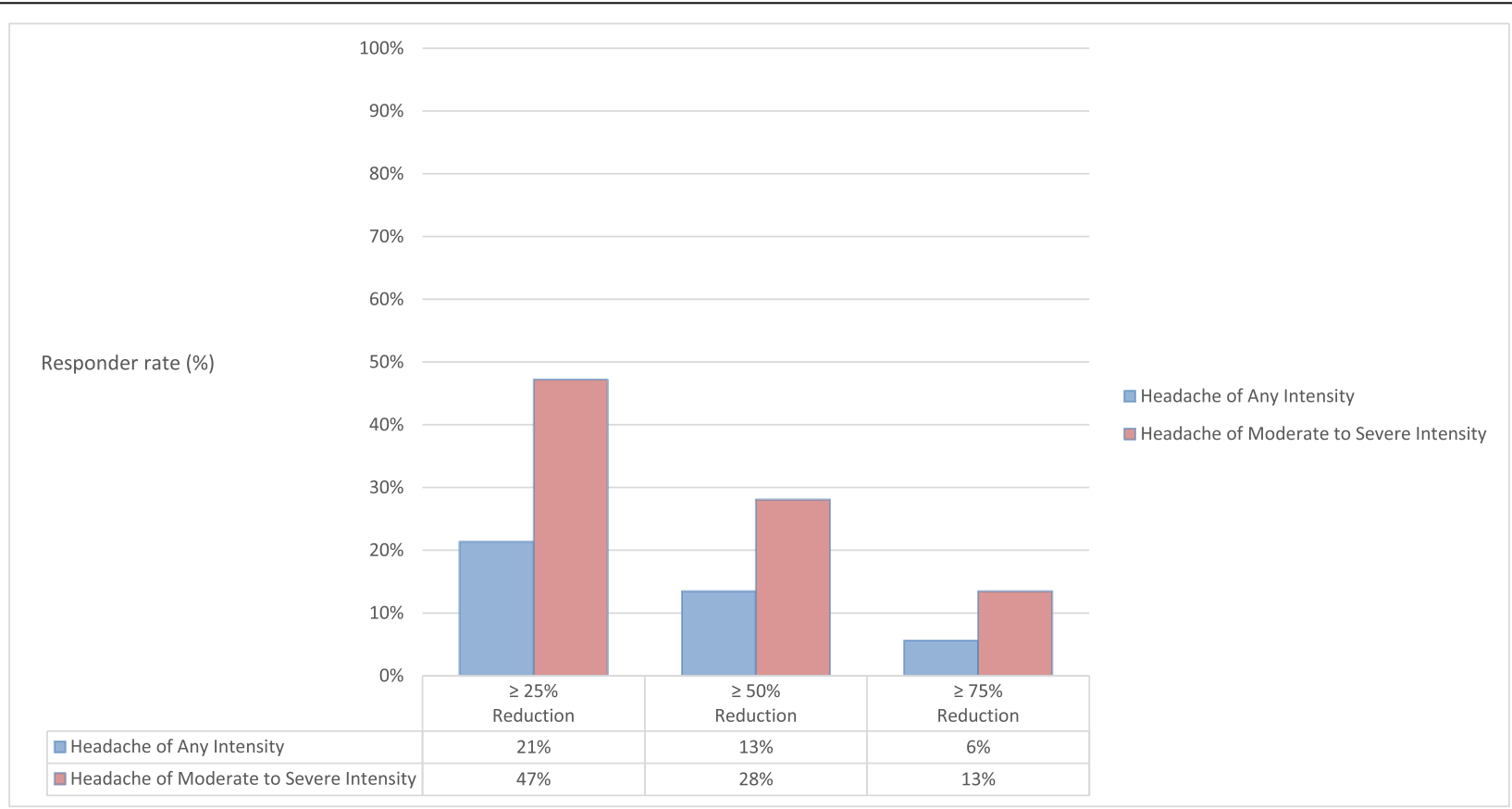

The responder rates were calculated as a percent reduction from baseline to week 9 through 12 in the number of headache days of moderate to severe intensity.

Fig. 2 Overview of 25\%,50\%, and 75\% Responder Rates. The responder rates were calculated as a percent reduction from baseline to week 9 through 12 in the number of headache days of moderate to severe intensity 
Table 3 Adverse Events during the 12-Week Open-Label Treatment Phase

\begin{tabular}{|c|c|}
\hline Adverse Events & Persistent PTH $(n=100)$ \\
\hline \multicolumn{2}{|l|}{ All Events } \\
\hline$\geq 1$ Adverse Event & 78 \\
\hline$\geq 1$ Treatment-Related Adverse Event & 38 \\
\hline$\geq 1$ Serious Adverse Event & 0 \\
\hline Any Adverse Event leading to Study Discontinuation & 2 \\
\hline \multicolumn{2}{|l|}{ Adverse Events occurring in $\geq \mathbf{2} \%$ of Patients ${ }^{\mathrm{a}}$} \\
\hline \multicolumn{2}{|l|}{ Injection-Site Reactions } \\
\hline Pain & 7 \\
\hline Erythema & 5 \\
\hline Hemorrhage & 3 \\
\hline Acid Reflux & 1 \\
\hline Constipation & 30 \\
\hline Diarrhea & 2 \\
\hline Dizziness & 9 \\
\hline Dry Mouth & 4 \\
\hline Fatigue & 5 \\
\hline Hot Flashes & 3 \\
\hline Influenza & 2 \\
\hline Irregular Menstruation & 3 \\
\hline Low Back Pain & 2 \\
\hline Nausea & 7 \\
\hline Palpitations & 2 \\
\hline Upper Abdominal Pain & 5 \\
\hline Worsened Headache & 8 \\
\hline
\end{tabular}

Data are reported as number of patients. If a patient had the same adverse event more than once, it was counted only once

reduction was $28 \%$. In those with at least 2 preventive treatment failures, the corresponding reduction was 3.0 headache days of moderate to severe intensity. Interestingly, one randomized clinical trial (RCT) found that erenumab yielded a reduction of 1.8 migraine days by week 9 through 12 in patients with high-frequency episodic migraine who had failed at least 2 preventive treatments [16]. Of note, the same study found that the corresponding reduction was only 0.2 migraine days in the placebo group [16]. Although our results cannot be directly compared with those of erenumab trials in migraine, it seems reasonable to draw some comparisons considering recently published data [6]. In a study of 91 patients with persistent PTH who had a migraine-like phenotype, the mean monthly number of migraine-like days was 14.5 [6]. Similarly, the mean monthly number of headache days of moderate to severe intensity was 15.7 at baseline in the present study population. Thus, it is likely that headache days of moderate to severe intensity largely reflect migraine-like headache days in patients with persistent PTH.
Overall, erenumab was well-tolerated, with most patients (89\%) receiving all three planned doses of erenumab. No safety concerns were found and only two patients discontinued the treatment regimen due to adverse events. The most frequently reported adverse events were constipation and injection-site reactions. Of note, nine of the 100 included patients reported recurrent episodes of constipation. However, this finding is of limited use as we did not record any data on the occurrence of occasional constipation prior to treatment with erenumab. In general, long-term data is needed to confirm the tolerability and safety of erenumab in individuals with persistent PTH.

Efficacy assessments are complicated by lack of placebo comparison. Thus, our findings should be interpreted with caution, although they provide context to observations made by clinicians who currently use erenumab as an off-label preventive treatment for individuals with persistent PTH. Moreover, it should be mentioned that 74 of the 100 included patients reported a history of preventive medication use. Of these, $86 \%$ 
had failed at least one preventive medication. It could be speculated that the placebo response is lower in those with history of preventive medication failure. Indeed, ample data from migraine trials with erenumab have consistently shown a lower placebo response in patients who reported previous failure of preventive medications [16-18]. As a migraine-like headache phenotype was present in 87 of the 100 included patients, it should be reasonable to assume that a similar placebo response is found in individuals with persistent PTH.

An important implication of the present study should be a future emphasis on use of standardized outcome measures and a requirement of subjects to fulfill the ICHD criteria for PTH [15]. This might facilitate comparative assessments and reduce heterogeneity between study populations. Similar conclusions were also made in a recent systematic review of preventive treatments for PTH [5]. Indeed, the authors were not able to infer efficacy of any preventive treatment due to an absence of placebo-controlled RCTs and a lack of high-quality open-label studies. In addition, efficacy outcomes had not been prospectively defined and varied between studies [19-21]. We would recommend that future intervention studies include prospectively defined outcomes measures that are documented using a headache diary. Furthermore, delineation of effective and well-tolerated preventive treatments requires intervention studies that apply a placebo-controlled RCT design. In this context, erenumab and other anti-CGRP monoclonal antibodies hold great promise as a growing body of evidence suggests CGRP involvement in the pathogenesis of PTH $[12,13]$. In particular, it would be very intriguing to assess whether treatment in the early phase following TBI could prevent development of persistent PTH at 3 months post-trauma.

\section{Limitations}

This study has several limitations. First, the study included an open-label design, without a placebo arm. As a result, it is difficult to interpret the efficacy and possible relatedness of an adverse event. Second, outcome measures were evaluated at a short-term follow-up of 3 months after the first dose of erenumab. Consequently, long-term observational studies are needed to adequately ascertain the efficacy and safety of erenumab. Third, assessment of previous treatment failures was done retrospectively using a semi-structured interview which may introduce recall bias. Fourth, efficacy outcome measures were evaluated using a headache diary in paper format, which does not allow monitoring of daily entries.

\section{Conclusions}

The present study suggests that erenumab might be a useful preventive treatment for persistent PTH. It appears that erenumab primarily reduces headache days of moderate to severe intensity, which often mimic the features of a migraine-like headache. As discontinuations due to adverse events were low, further research is much needed to assess the effectiveness of erenumab against placebo as well as other preventive medications.

\section{Abbreviations}

PTH: Post-traumatic headache; TBI: Traumatic brain injury; CGRP: Calcitonin gene-related peptide; ICHD-3: International Classification of Headache Disorders 3rd edition; TTH: Tension-type headache; HIT-6: Headache Impact Test; RCT: Randomized clinical trial

\section{Acknowledgements}

Not applicable.

\section{Authors' contributions}

Håkan Ashina: Study concept and design, acquisition of data, analysis (including statistical analyses) and interpretation, drafting the manuscript. Afrim Iljazi: Acquisition of data and critical revision of the manuscript for important intellectual content. Haidar Muhsen Al-Khazali: Acquisition of data and critical revision of the manuscript for important intellectual content. Anna Kristina Eigenbrodt: Acquisition of data and critical revision of the manuscript for important intellectual content. Eigil Lindekilde Larsen: Acquisition of data and critical revision of the manuscript for important intellectual content. Amalie Middelboe Andersen: Acquisition of data and critical revision of the manuscript for important intellectual content. Kevin John Hansen: Acquisition of data and critical revision of the manuscript for important intellectual content. Karoline Bendix Bräuner: Acquisition of data and critical revision of the manuscript for important intellectual content. Thomas Mørch-Jessen: Acquisition of data and critical revision of the manuscript for important intellectual content. Basit Chaudhry: Acquisition of data and critical revision of the manuscript for important intellectual content. Sonja Antic: Critical revision of the manuscript for important intellectual content. Casper Emil Christensen: Analysis (including statistical analyses) and interpretation, critical revision of the manuscript for important intellectual content. Faisal Mohammad Amin: Critical revision of the manuscript for important intellectual content. Messoud Ashina: Study concept and design, critical revision of the manuscript for important intellectual content. Henrik Winter Schytz: Study concept and design, critical revision of the manuscript for important intellectual content, supervision. The authors read and approved the final manuscript.

\section{Funding}

The authors disclosed receipt of the following financial support for the research, authorship, and/or publication of this Article: The study was supported by an investigator-initiated research grant from Novartis Healthcare A/S who also provided the study drug.

\section{Availability of data and materials}

Qualified researchers can request access to patient-level data and related study documents, including the study protocol. Patient-level data will be deidentified and study documents will be redacted to protect the privacy of trial participants.

\section{Ethics approval and consent to participate}

Informed written consent was obtained from each patient before conducting any study-related procedures. The study protocol was reviewed by the Regional Health Research Ethics Committee of the Capital Region of Denmark (identifier: H-18050498).

\section{Consent for publication}

Not applicable.

\section{Competing interests}

The authors declared the following potential conflicts of interest with respect to the research, authorship and/or publication of this article: Dr. Casper Emil Christensen has lectured and been a scientific advisor for Teva. Dr. Messoud Ashina has received personal fees from Alder BioPharmaceuticals, Allergan, Amgen, Eli Lilly, Novartis, and Teva. Dr. 
Messoud Ashina has been or currently is a principal investigator on clinical trials for Alder, Amgen, electroCore, Novartis, and Teva. Dr. Messoud Ashina also serves as an Associate Editor of Cephalalgia, Associate Editor of Headache, and Co-Editor of the Journal of Headache and Pain. Dr. Messoud Ashina reports research grants from Lundbeck Foundation, Novo Nordisk Foundation, and Research Foundation of the Capital Region of Denmark. Dr. Faisal Mohammad Amin has received personal fees from Eli Lilly, Novartis and Teva. Dr. Faisal Mohammad Amin is currently a principal investigator on clinical trials for Novartis and Teva. Dr. Faisal Mohammad Amin also serves as an Associate Editor of Headache Medicine. Dr. Henrik Winther Schytz has received speaking fees from Novartis and Teva. The other authors declare no conflicts of interest.

\section{Author details}

'Danish Headache Center, Department of Neurology, Rigshospitalet Glostrup, Faculty of Health and Medical Sciences, University of Copenhagen, Valdemar Hansens Vej 5, DK-2600 Glostrup, Denmark. ${ }^{2}$ Department of Neurology, Aarhus University Hospital, Aarhus, Denmark.

Received: 30 April 2020 Accepted: 27 May 2020

Published online: 03 June 2020

\section{References}

1. Nampiaparampil DE (2008) Prevalence of chronic pain after traumatic brain injury: a systematic review. JAMA 300(6):711-719

2. van der Naalt J, Timmerman ME, de Koning ME, van der Horn HJ, Scheenen ME, Jacobs B et al (2017) Early predictors of outcome after mild traumatic brain injury (UPFRONT): an observational cohort study. Lancet Neurol 16(7): 532-540

3. Rasmussen BK, Olesen J (1992) Symptomatic and nonsymptomatic headaches in a general population. Neurology 42(6):1225-1231

4. Ashina H, Porreca F, Anderson T, Amin FM, Ashina M, Schytz HW et al (2019) Post-traumatic headache: epidemiology and pathophysiological insights. Nat Rev Neurol 15(10):607-617

5. Larsen EL, Ashina H, Iljazi A, Al-Khazali HM, Seem K, Ashina M et al (2019) Acute and preventive pharmacological treatment of post-traumatic headache: a systematic review. J Headache Pain 20(1):98

6. Ashina H, lljazi A, Al-Khazali HM, Ashina S, Jensen RH, Amin FM, et al (2020) Persistent post-traumatic headache attributed to mild traumatic brain injury: deep phenotyping and treatment patterns. Cephalalgia 40(6):554-64

7. Goadsby PJ, Reuter U, Hallström Y, Broessner G, Bonner JH, Zhang F et al (2017) A controlled trial of Erenumab for episodic migraine. N Engl J Med 377(22):2123-2132

8. Silberstein SD, Dodick DW, Bigal ME, Yeung PP, Goadsby PJ, Blankenbiller T et al (2017) Fremanezumab for the preventive treatment of chronic migraine. N Engl J Med 377(22):2113-2122

9. Stauffer VL, Dodick DW, Zhang Q, Carter JN, Ailani J, Conley RR (2018) Evaluation of Galcanezumab for the prevention of episodic migraine: the EVOLVE-1 randomized clinical trial. JAMA Neurol 75(9):1080-1088

10. Ashina M, Saper J, Cady R, Schaeffler BA, Biondi DM, Hirman J et al (2020) Eptinezumab in episodic migraine: a randomized, double-blind, placebocontrolled study (PROMISE-1). Cephalalgia 40(3):241-254

11. Charles A, Pozo-Rosich P (2019) Targeting calcitonin gene-related peptide: a new era in migraine therapy. Lancet 394(10210):1765-1774

12. Navratilova E, Rau J, Oyarzo J, Tien J, Mackenzie K, Stratton J et al (2019) CGRP-dependent and independent mechanisms of acute and persistent post-traumatic headache following mild traumatic brain injury in mice. Cephalalgia 39(14):1762-1775

13. Bree D, Levy D (2018) Development of CGRP-dependent pain and headache related Behaviours in a rat model of concussion: implications for mechanisms of post-traumatic headache. Cephalalgia 38(2):246-258

14. World Medical Association (2013) World medical association declaration of Helsinki: ethical principles for medical research involving human subjects. JAMA 310(20):2191-2194

15. Classification Committee of the International Headache Society (2018) The international classification of headache disorders (ICHD), 3rd edition. Cephalalgia 38(1):1-211

16. Reuter U, Goadsby PJ, Lanteri-Minet M, Wen S, Hours-Zesiger P, Ferrari MD et al (2018) Efficacy and tolerability of Erenumab in patients with episodic migraine in whom two-to-four previous preventive treatments were unsuccessful: a randomised, double-blind, placebo-controlled, phase 3b study. Lancet 392(10161):2280-2287

17. Goadsby PJ, Paemeleire K, Broessner G, Brandes J, Klatt J, Zhang F (2019) Efficacy and safety of Erenumab (AMG334) in episodic migraine patients with prior preventive treatment failure: a subgroup analysis of a randomized, double-blind, placebo-controlled study. Cephalalgia 39(7):817826

18. Ashina M, Tepper S, Brandes JL, Reuter U, Boudreau G, Dolezil D (2018) Efficacy and safety of Erenumab (AMG334) in chronic migraine patients with prior preventive treatment failure: a subgroup analysis of a randomized, double-blind, placebo-controlled study. Cephalalgia 38(10): 1611-1621

19. Cushman DM, Borowski L, Hansen C, Hendrick J, Bushman T, Teramoto M (2019) Gabapentin and Tricyclics in the treatment of post-concussive headache, a retrospective cohort study. Headache. 59(3):371-382

20. Kuczynski A, Crawford S, Bodell L, Dewey D, Barlow KM (2013) Characteristics of post-traumatic headaches in children following mild traumatic brain injury and their response to treatment: a prospective cohort. Dev Med Child Neurol 55(7):636-641

21. Erickson JC (2011) Treatment outcomes of chronic post-traumatic headaches after mild head trauma in us soldiers: an observational study. Headache. 51(6):932-944

\section{Publisher's Note}

Springer Nature remains neutral with regard to jurisdictional claims in published maps and institutional affiliations.
Ready to submit your research? Choose BMC and benefit from:

- fast, convenient online submission

- thorough peer review by experienced researchers in your field

- rapid publication on acceptance

- support for research data, including large and complex data types

- gold Open Access which fosters wider collaboration and increased citations

- maximum visibility for your research: over $100 \mathrm{M}$ website views per year

At BMC, research is always in progress.

Learn more biomedcentral.com/submissions 